\title{
The role of IL-8 in the immune response associated to cancer development
}

\author{
Manuela Gonzalez-Aparicio ${ }^{1}$ and Carlos Alfaro ${ }^{2 *}$ \\ ${ }^{1}$ Gene Therapy Program, Fundación para la Investigación Médica Aplicada, CIMA, Universidad de Navarra, Instituto de Investigación Sanitaria de Navarra \\ (IdiSNA), Av. Pio XII 55, Pamplona 31008, Spain \\ ${ }^{2}$ Oncohematology Research Group, Navarrabiomed, Complejo Hospitalario de Navarra, Universidad Pública de Navarra, Instituto de Investigación Sanitaria de \\ Navarra (IdiSNA). Irunlarrea 3, 31008 Pamplona, Spain
}

\section{Introduction}

Among the biological mediators that synthesize the cells of the immune system, in response to pathogens or other signals, we find chemokines. It is a particular type of cytokines whose main function is not the communication between different immune cells but chemotaxis, that is, the attraction of leukocytes to a specific place in the body.

The chemotactic cytokines can be produced by several cell types such as monocytes, macrophages, $\mathrm{T}$ lymphocytes, fibroblasts and endothelial cells during the inflammation of a tissue, to promote the development of certain immune cells, to maintain a correct lymphocyte circulation or to attract a certain cell type to a specific organ. Specifically the interleukins (cytokines secreted by leukocytes) are regulated by the factor NF-kappa B, which also regulates the expression of genes in innate and acquired immunity.

To exert their function, once synthesized, the interleukins bind to specific receptors that are found in the membrane of the target cells, where they are recognized and interpreted as a "call" or "recruitment" signal. The attraction towards the concrete tissue is realized thanks to what is known as a "chemotactic gradient", that is, a gradient of concentration of the chemokine. In this way cells are attracted from the place of lowest concentration of this molecule to the place where it is in the highest concentration.

\section{Role of the IL-8}

Among the main chemokines, is IL-8 (or CXCL-8). It is known especially for being able to promote the adhesion of the monocytes and neutrophils that are in the blood circulation to the endothelial cells that form the blood vessels, helping them to pass from the blood to the inflamed tissue, so that they can exercise their action. It is known, for example, that this molecule is essential in a bacterial infection, to attract neutrophils to the site of infection and thus eliminate bacteria.

However, it should be noted that this cytokine has also been linked to different pathologies. For example, it is known that it is involved in

Copyright: (C)2018 Gonzalez-Aparicio M. This is an open-access article distributed under the terms of the Creative Commons Attribution License, which permits unrestricted use, distribution, and reproduction in any medium, provided the original author and source are credited. the infiltration of monocytes in atheromatous plaques, contributing to the process of atherosclerosis. It also participates in the inflammatory process characteristic of rheumatoid arthritis. Similarly it is known that this molecule promotes angiogenesis in endothelial cells, facilitating the development of cancer.

IL-8 stimulates genes that inhibit the activity of proteins called suppressors of cytokine signaling (SOCS). As a consequence of this suppression, STAT-3 transcription factors and the activity of macrophages associated with tumors increase, favoring the migration of tumor cells. The activation of STAT-3 also promotes the growth and survival of tumor cells. This is because it stimulates cell cycle regulatory proteins such as cyclins D1/D2 and activates anti-apoptotic proteins such as: myeloid cell leukemia 1 (MCL-1), B-cell lymphomaextra large (Bcl-XL) and X-linked protein (XIAP). Also one of the roles of IL-8 is to inhibit the expression of p53 tumor suppressor genes and induce angiogenesis by activating vascular endothelial growth factor (VEGF).

\section{Diagnostic capacity of IL-8}

A tumor biomarker is a substance whose presence indicates a change in cellular processes. Biomarkers may be substances normally produced by normal cells, but which modify their concentration consistently, against a disease or in response to a neoplastic process or produced by the cancer cells themselves. Most biomarkers are proteins and can be obtained from biological samples such as serum, urine, cerebrospinal fluid or saliva.

Recently, clear evidences have appeared that indicate that IL-8 is a potentially useful biomarker to monitor changes in tumor burden following anticancer therapy, and has prognostic significance. It is based on that serum concentrations of IL-8 accurately reflect the viability of tumor cells and correlate with tumor burden in a variety of solid human tumors. This change in values is possible to check at the beginning and after antitumor treatment, so it could be a useful platform for evaluating antiangiogenic and immunotherapeutic agents.

*Correspondence to: Carlos Alfaro, Oncohematology Research Group, Navarrabiomed, Complejo Hospitalario de Navarra, Universidad Pública de Navarra, Instituto de Investigación Sanitaria de Navarra (IdiSNA), Irunlarrea 3. 31008 Pamplona, Spain, E-mail: calfale@unav.es

Received: December 07, 2018; Accepted: December 14, 2018; Published: December 17, 2018 